\title{
Aplicación de force concept inventory en América Latina para la evaluación de la comprensión de los conceptos básicos de mecánica a nivel universitario
}

\author{
Irina Artamónova ${ }^{a}$, Julio César Mosquera-Mosquera ${ }^{a} \&$ José Daniel Mosquera-Artamónov ${ }^{b}$ \\ a Facultad de Ciencias Básicas, Universidad del Quindio,Armenia,Colombia,artiri@gmail.com, jcmosquera@uniquindio.edu.co \\ b Posgrado en Ingeniería de Sistemas, Universidad Autónoma de Nuevo León, Monterrey, México, xoce15@ingenieros.com
}

\begin{abstract}
Resumen - La baja comprensión de los conceptos básicos de la mecánica newtoniana dificulta el desempeño de los estudiantes universitarios, tanto en cursos de mecánica como en los cursos subsiguientes que exigen el uso de tales conceptos fundamentales. En este artículo se utiliza la información de varias investigaciones realizadas en América Latina, Estados Unidos y España en ambientes universitarios en los cuales se utilizó el test Force Concept Inventory para la evaluación de la comprensión de los conceptos básicos de la mecánica newtoniana. Se muestra una forma fácil de comparar los resultados promedios de varios grupos en pre-test, post-test y la eficiencia didáctica del curso con otros grupos incluso con otras universidades de otros países. Este artículo ofrece, a la comunidad de educadores latinoamericanos, un marco teórico propio para hacer comparaciones entre diferentes propuestas pedagógicas, lo que permite el mejoramiento de planes de estudio y la evaluación de necesidad de cambios curriculares.
\end{abstract}

Palabras Clave - enseñanza de la física, técnicas de evaluación, test FCI, test conceptuales, mecánica newtoniana.

Recibido: 16 agosto de 2016. Revisado: 20 de septiembre de 2016. Aceptado:6 de octubre de 2016.

\section{Application of force concept inventory in Latin America for evaluation the understanding of the basic concepts of mechanics at the university level}

\begin{abstract}
The low understanding of these issues hinders the students performance in mechanics university's courses as well in subsequent courses that require the use of such fundamental concepts. Using information from various investigations in Latin America, USA and Spain in university environments in which the test Force Concept Inventory for assessment of understanding of the basic concepts of Newtonian mechanics were used. This article offers to Latin American educator's community, its own theoretical framework to evaluate the efficiency of the methodology used in Physics teaching - learning process and to make comparisons between different teaching methodologies, enabling the curricula improvement and assessment of the needs of curricular changes. This theoretical framework based on research conducted in Latin America university environments. On these researches the Force Concept Inventory test were used, which allows not only knowing the scores achieved by students in pre-test and post-test but the course teaching efficiency.
\end{abstract}

Keywords - research in physics education, techniques of testing; test FCI, conceptual test, Newtonian mechanics

\section{Introducción}

El proceso enseñanza - aprendizaje de la física a nivel universitario está acompañado de serias dificultades conceptuales. Los estudios realizados evidencian que conceptos físicos como masa [1], fuerza [2-6], inercia [7] y en general conceptos ligados a la concepción de las leyes de Newton, que son el fundamento de la física mecánica, no son bien asimilados por los estudiantes. La baja comprensión de estos temas dificulta el desempeño de los estudiantes de diferentes carreras de ciencias o de ingenierías [1,8-10]. Las dificultades se presentan tanto en cursos de mecánica como en los cursos subsiguientes que exigen el uso de tales conceptos fundamentales. Existen pruebas surgidas de investigaciones que los alumnos que han hecho la experiencia de la resolución de problemas cualitativos resuelven con más facilidad los problemas cuantitativos, que aquellos que han pasado más tiempo resolviendo los problemas tradicionales $[11,12]$. Los errores conceptuales son persistentes en todos los niveles de formación y son difíciles de erradicar [13]. En muchos casos no es posible continuar la formación de los estudiantes en una carrera determinada, lo cual conduce a problemas de deserción estudiantil. Muchos docentes se dedican a buscar que los estudiantes desarrollen habilidades matemáticas en la resolución de problemas extraídos de fenómenos físicos de la mecánica, y poco enfatizan en el desarrollo conceptual del alumno. El estudio de Aguilar y Durán [14] en diferentes carreras de ingenierías de la Universidad Industrial de Santander de Colombia corrobora los resultados encontrados en contextos diferentes al de educación en ingeniería, pero relacionados con el aprendizaje de la fisica, donde se sugiere la existencia de una relación de dependencia entre el contenido (dominio conceptual) y el nivel cognitivo (competencia) desarrollado por los estudiantes". El análisis multidimensional realizado en la investigación de Kahan [15] ha demostrado que los resultados de los parciales del segundo semestre están fuertemente correlacionados con el aprendizaje de los

Como citar este artículo: Artamónova, I., Mosquera-Mosquera, J. C. \& Mosquera-Artamónov, J. D.. Aplicación de force concept inventory en América Latina para la evaluación de la comprensión de los conceptos básicos de mecánica a nivel universitario. Revista Educación en Ingeniería 12 (23) 56-63, Febrero, 2017. 
conceptos básicos de mecánica involucrados, demostrando la importancia del aprendizaje conceptual de la física. Pese a la importancia de aprendizaje conceptual las evaluaciones típicas en los cursos de mecánica en algunos universidades no miden el desarrollo conceptual de los estudiantes si no su capacidad en resolver problemas, lo que genera un vacío en el proceso enseñanza - aprendizaje.

En Estados Unidos en los años 90 fueron diseñadas varias pruebas para evaluar el manejo de los conceptos básicos de mecánica newtoniana por los estudiantes universitarios. Una de estas pruebas es el test denominado Force Concept Inventory (FCI) - que es un Cuestionario Sobre el Concepto de Fuerza publicado en 1992 por David Hestenes, Malcolm Wells y Gregg Swackhamer [16]. Este test tuvo un gran reconocimiento en el ámbito de la investigación educativa relacionada con la enseñanza de la Física y en particular de la Mecánica en Estados Unidos. Versiones del FCI (1995) fueron traducidas a diversas lenguas con la intención de facilitar su difusión en otras culturas y países. Con la estimación de Hestenes y Halloun [17] el test FCI fue aplicado a 10000 alumnos y por más de 100 profesores. Hake [18] en su artículo habla de una aplicación del test a 6542 estudiantes. Cronch y Mazur [19] aplicaron este test a una población de 1624 alumnos con una metodología de enseñanza denominada Peer Instruction. En las investigaciones de Henderson [20] el test se aplicó a una población de 2178 alumnos. Cabalero y otros [21] aplicaron FCI a 5000 estudiantes. Fagen, Crouch y Mazur [22] dicen que más de 500 profesores que aplicaron la metodología Peer Instruction administraron FCI en sus cursos para evaluar los resultados de los estudiantes.

Para el año 2008 Covián y Celemín hacen un análisis de la utilización del test FCI en España [23], donde se aplicó la versión del test FCI traducida al español por Celemín y que contenía 29 preguntas en total en años anteriores. En esta investigación fueron encuestados 1300 alumnos en el periodo comprendido entre los cursos 1992-1993 y 2000-2001 en 7 distintas universidades de España. De esta forma el test FCI se convirtió en la herramienta más utilizada para determinar los conocimientos de los estudiantes y para evaluación de la eficiencia didáctica de diferentes cursos y metodologías en temas de Mecánica Newtoniana. Actualmente se pueden encontrar versiones del test FCI en la página web de American Modeling Teachers Association en inglés, español, alemán, francés, italiano, $\mathrm{y}$ en total en 21 idiomas (http://modelinginstruction.org/researchers/evaluation-

instruments/fci-and-mbt). Gracias a estas numerosas investigaciones es posible evaluar simultáneamente diferentes propuestas académicas tomando como base el desarrollo conceptual de los educandos.

\section{Aplicaciones del test FCI}

\subsection{Determinar el nivel de conocimientos de Mecánica}

En este caso el porcentaje de respuestas correctas del test FCI que se calcula para cada estudiante constituye una medida válida del nivel de conocimientos de Mecánica (Hestenes et al., [16]; Hake, [18]; Henderson, [20]). Este porcentaje se calcula tanto al principio $(\%<$ pre $>)$, como al final del curso de mecánica $(\%<$ post $>)$. Para Hestenes y Halloun [17] un nivel mínimo de conocimientos y de comprensión de la Mecánica válido se sitúa en el $60 \%$ de respuestas correctas del FCI (18 respuestas correctas de 30 preguntas); el nivel exigido en el contexto español se considera situado en el 50\% (15 respuestas correctas de 30 preguntas) (Covián y Celemín, [23]). Hestenes y Halloun [17] proponen que el límite a partir del cual se da un nivel de conocimientos y de comprensión de la Mecánica comparable a la concepción newtoniana (nivel de master) se sitúa en el $85 \%$ de respuestas correctas del FCI $(25,5$ en promedio de respuestas correctas de 30 preguntas).

\subsection{Calcular la ganancia individual de aprendizaje (factor g de Hake)}

Es usual que el grupo de entrada a un determinado curso sea muy heterogéneo y en general así mismo son los resultados finales. Si se realizan dos pruebas, una antes de dictar el tema y otra después que el tema ya ha sido presentado a los estudiantes, sus resultados se reportan como un valor de g de Hake que es un número denominado "ganancia normalizada" y que es la razón del aumento entre una prueba preliminar (pre) y un prueba final (post) respecto del máximo aumento posible (ec. (1)):

$$
\mathrm{g}=\frac{\%<\text { post }>-\%<\text { pre }>}{100 \%-\%<\text { pre }>}
$$

Utilizar la ganancia normalizada permite evaluar el progreso de cada estudiante evitando el problema de comparar entre estudiantes que empiezan un curso mejor preparados que otros. Tal información sirve para elaborar planes de complemento didáctico para aquellos estudiantes que aunque no han alcanzado un alto nivel de conocimientos, si están mejor motivados para continuar la profundización en la temática estudiada.

Hake [18] considera tres rangos de ganancia normalizada:

- $\quad g$ alto - Cuando el resultado obtenido para $g$ es $g>0,7$;

- $g$ medio - Cuando el resultado obtenido para $g$ está en el rango $0,3<\mathrm{g}<0,7$;

- $g$ bajo - Cuando el resultado obtenido para $g$ es $g<0,3$.

\subsection{Calcular la eficiencia didáctica del curso}

La Eficiencia didáctica (ec. (2)) es definida por Covián y Celemín [23] como "el incremento relativo de respuestas correctas entre la primera y la segunda aplicaciones del FCI, respecto de la mayor mejora posible, o eficiencia didáctica, que representa la influencia del proceso enseñanza-aprendizaje en el nivel de conocimiento de la percepción newtoniana.

$$
\begin{aligned}
& \text { Eficienciadidáctica }=\bar{\Delta}_{r e l .} B= \\
& =\frac{\bar{B}_{2^{a} \text { aplic del } F C I}-\bar{B}_{1^{a} \text { aplicdel } F C I}}{100-\bar{B}_{1^{a} \text { aplic del } F C I}}, \%
\end{aligned}
$$


El valor B aquí es la media del porcentaje de respuestas correctas del FCI para todo el grupo en Pre-test o Post-test.

La eficiencia didáctica promedia en poblaciones estadounidenses para cursos con metodología tradicional de aprendizaje fue de $23 \%$ y para los cursos con metodología interactiva de aprendizaje fue de 48\% (Hake, [18]). Es claro que este factor no busca evaluar al alumno mismo, sino más bien el proceso enseñanza - aprendizaje y las estrategias didácticas del docente.

\section{El objetivo y la metodología de investigación}

El objetivo de la presente investigación fue recolectar los resultados de aplicación del test FCI en diferentes grupos de estudiantes de ingenierías o de ciencias, incluso en diferentes países $\mathrm{y}$ hacer una hipótesis sobre la relación entre los resultados promedios de pre-test FCI y los resultados promedios de post-test FCI para un grupo de estudiantes en un caso concreto. Ya que el test FCI es ampliamente utilizado, incluso en diferentes países durante los últimos 30 años, esto permite hacer una sola gráfica para muestras de diferentes países donde el test FCI fue aplicado a nivel universitario y hacer ciertas inferencias sobre la información de los Pre-Test, Post-Test y la eficiencia didáctica al mismo tiempo. Se hace énfasis en la propuesta pedagógica que fue empleada en cada grupo de estudiantes con el propósito de resaltar las metodologías más exitosas desarrolladas por los docentes. Todo esto con el propósito a ayudar a cada docente, después de aplicar el test FCI, evaluar qué tan bien resultó el proceso de aprendizaje de los conceptos básicos de mecánica, ya que simultáneamente con el análisis de los resultados de Post-Test FCI se puede revisar la eficiencia didáctica del curso y comparar con los resultados obtenidos en otros grupos en otros lugares.

\section{Análisis de resultados de investigaciones en América Latina}

En la Fig. 1 se presenta gráficamente la relación existente entre los promedios de Post-test FCI para diferentes grupos de estudiantes de diferentes países, respecto a los promedios del Pre-test FCI. Mayor información sobre cada grupo se puede obtener de la Tabla 1, y de las referencias bibliográficas que están en la primera columna de esta misma tabla. La información relacionada con Estados Unidos fue tomada de la tabla 3 de Covián y Celemín ([23], p.28).

Se puede observar que todos los grupos pueden ser divididos en sub-grupos de acuerdo al promedio del Pre-test que se obtuvo. Cada sub-grupo está encerrado en una elipse. La línea roja representa casos cuando el promedio en Post- test es estadísticamente el mismo que en Pre-test, lo que indica que no hubo aprendizaje conceptual y lo cual se refleja en valores $g$ de Hake iguales a cero. Línea verde corresponde a la ec. (3):

\section{Post-test $=0,7^{*}$ Pre-test $\mathrm{FCl}+30$}

Esta línea verde representa los casos cuando la eficiencia didáctica es igual a $30 \%$, con lo cual los puntos que están en medio de las líneas roja y verde con eficiencia didáctica $0 \%<\mathrm{g}$ $<30 \%$, son casos considerados como de eficiencia didáctica baja. Línea azul representa la eficiencia didáctica igual a 70\% y corresponde a la ec. (4):

Post-test $=0,3 *$ Pre-test $\mathrm{FCl}+70$

Los puntos que están entre la línea azul y verde son grupos con la eficiencia didáctica media. Y los grupos de eficiencia didáctica alta estarían por encima de la línea azul (Fig.1).

En el primer grupo señalado en la gráfica con la elipse de la izquierda (Fig. 1), los promedios de Pre-test FCI varían entre 18,8\% y $27,7 \%$. En este grupo la mayoría de los estudiantes no tienen conocimientos de mecánica newtoniana o respondieron el test FCI de forma aleatoria. En muchas universidades no hay elección de los estudiantes que ingresan a una carrera por sus conocimientos en física, si no que tienen otros criterios de ingreso. En este primer grupo en Post-test FCI los promedios que alcanzan los estudiantes varían desde $26,5 \%$ hasta $59,7 \%$. En total la variación es alrededor de $33,2 \%$. Los promedios más bajos en Post-test FCI corresponden a los grupos de control donde fue aplicada la metodología tradicional de aprendizaje por recepción- transmisión de conocimientos en Argentina, Colombia y México. Muchos cuadrados rojos corresponden a una sola universidad en Colombia pero a diferentes programas y metodologías aplicadas en la Universidad del Quindío. Solo un grupo de Colombia de esta categoría de "novatos" está por encima de la línea verde que significa que obtuvo la eficiencia didáctica mayor de $30 \%$, este fue el grupo donde fue aplicada la metodología 4MAT (Artamónova, [24]).

Igualmente se observan dos círculos para Argentina (Mercado, [25]), uno corresponde a metodología tradicional y otro con el promedio 51,0\% de Post-test FCI a metodología experimental que alcanzaron los estudiantes de la Facultad de Ingeniería y Ciencias Agropecuarias de la Universidad Nacional de San Luis de Argentina [25]. La metodología experimental aplicada en esta universidad fue nombrada IDEA (Beichner, [26]) y consiste en los siguientes pasos: Informarse / Desarrollar un Plan / Ejecutar el plan / Aprender de sus esfuerzos. La estrategia IDEA se implementó en un marco de aprendizaje colaborativo (Panitz, [27]) donde se desarrolló la interacción entre los compañeros de clase. Esta es una herramienta de gran valor pedagógico basada en discusiones grupales que aceleran el intercambio de argumentos y razonamientos hasta alcanzar el consenso necesario. Los problemas para resolver en el grupo con IDEA eran de los problemas "ricos en contexto".

Los problemas ricos en contexto (Heller, [28,29]) presentan algunos rasgos particulares: son fundamentalmente relacionados con las situaciones cotidianas; no son fáciles de resolver de manera individual. Por lo general, tienen o exceso o insuficiencia de información, así como pasa con los problemas reales. Adicionalmente éstos contienen palabras como "usted" para que el estudiante pueda sentirse implicado en el problema. El resultado de esta metodología es que los estudiantes puedan resolver más problemas difíciles que los estudiantes con el enfoque tradicional y parecen mejorar las habilidades de razonamiento $\mathrm{y}$ desarrollar una profunda comprensión de los conceptos y principios (Leonard, [30]). 


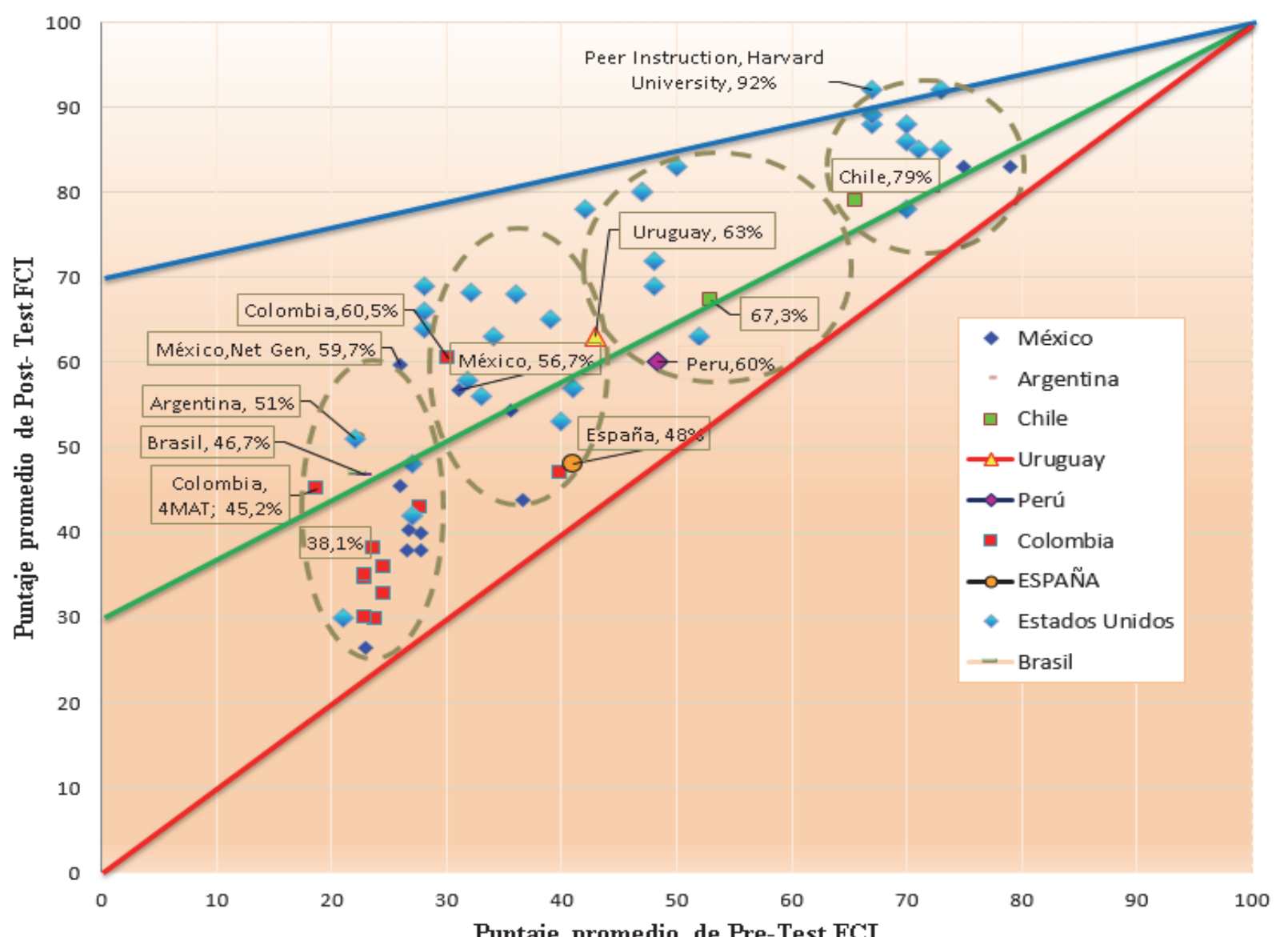

Figura 1. Relación Pre-test FCI - Post-test FCI para diferentes grupos de estudiantes de diferentes países.

Fuente: Los autores.

Los estudiantes de licenciatura en Química de la Universidad Tecnológica Federal de Paraná de Brasil (Goya y Laburú, [31]) lograron llegar a 46,9\% en Post-test FCI. La metodología utilizada en esta universidad fue - actividad de investigación multimodal representacional - AIM. En la práctica de la enseñanza de las ciencias, esto equivale a someter a los estudiantes a modos diferentes de representación, ya sea oral, gráficos, tablas, figurativa, esquemática, fotográfica, analógica, metafóricas, kinestésica como experimentos (3-D), modelos (3-D) y los gestos, representaciones matemáticas, fílmicas, entre otros. Para desarrollar una actividad de investigación multimodal (AIM) podrían unirse la autonomía de la metodología de la investigación con el aprendizaje a profundización multimodal y múltiples representaciones (Laburú, [32]). Los procedimientos consistieron en momentos o pasos flexibles de la siguiente presentación: I) Fenómeno; II) Problema; III) Hipótesis; IV) Plan de Trabajo; V) Análisis; Vi) Conclusión; VII) Comunicación de los resultados a través de forma multimodal $\mathrm{y}$ de múltiples representaciones. Entendiendo la comunicación de los resultados como un paso adicional a la "Conclusión", de modo que el grupo de estudiantes después de resolver un problema complementa la fijación del aprendizaje a través de la comunicación de los resultados a otros grupos. La mayoría de los estudiantes con los cuales se utilizó AIM considera la actividad de investigación multimodal (AIM) mejor que la enseñanza tradicional, sobre todo en la motivación y comprensión de los contenidos [31].

Otro grupo de los estudiantes de Programa de Química de la Universidad del Quindío de Colombia logró llegar a 45,2\% en Post test FCI empezando con apenas 18,8\% de respuestas correctas en Pre-test [24]. La metodología utilizada en este grupo fue 4MAT que toma en cuenta los 4 estilos de aprendizaje de los estudiantes. El grupo de Ciencias Básicas e Ingenierías de la Universidad Autónoma Metropolitana Iztapalapa de México alcanzó resultados similares a la Universidad del Quindío con $45,4 \%$ en promedio de Post-test FCI, pero tiene una eficiencia didáctica baja puesto que sus estudiantes en Pretest FCI obtuvieron un promedio de $26 \%$. Esto último es un buen ejemplo del poderío comparativo del FCI. Gómez [33] propone en dos universidades mexicanas un modelo de enseñanza-aprendizaje que parte de una conceptuación rigurosa de las leyes de Newton y se fundamenta en aportaciones de la neurociencia aplicadas a la educación. Aunado a ello se toman en consideración las características de la llamada Generación Net o, simplemente Net Gen en el diseño de un nuevo modelo de la enseñanza-aprendizaje donde se aprovecharon las TICs con fines educativos llegando a los resultados de 59,6\% de respuestas correctas en el Post test FCI. Este fue el mejor resultado en primer grupo de "novatos" donde se alcanzó más alta eficiencia didáctica de $48 \%$. 
En la segunda elipse de la Fig. 1 están los grupos que tienen mejores criterios de selección de los estudiantes para entrar a la universidad. Los promedios de Pre-test en este grupo varían desde $30,2 \%$ hasta $41,0 \%$. En Post-test los promedios varían desde $43,9 \%$ hasta $68,2 \%$. El círculo color naranja corresponde a la población de estudiantes universitarios de España con Pretest de $41 \%$ y Post-test de $48 \%$, con una eficiencia didáctica de $12 \%$. El grupo de los estudiantes del Programa de Física de la Universidad del Quindío se comporta casi como la población universitaria española [23]. Las poblaciones universitarias de Estados Unidos están en la segunda elipse y son los que sacaron el mayor promedio en Post-test - 68,2\%. El grupo de estudiantes de la Licenciatura en Ciencias Naturales y Educación Ambiental de la Universidad de Antioquia de Colombia, que sacó 60,5\% en Post-test (López, [34]), logró resultados un poco mejores que la población de los estudiantes de Estados Unidos que obtuvieron un promedio en Post- test FCI de 57,9\% [23]. Casi los mismos resultados - 56,7\% se alcanzaron en dos universidades de Guadalajara, Jalisco, México con estudiantes de carreras de ingeniería y ciencias en el grupo experimental (Gómez, [8]). El grupo de la Universidad de Antioquia [34] presenta hasta ahora los mejores resultados reportados en literatura para Colombia.

En la tercera y cuarta elipses de la Fig.1 están los grupos avanzados. Son grupos de las universidades de alto prestigio cada uno en su país, así como por ejemplo, Harvard University de Estados Unidos. De todas las universidades latinoamericanos que se encontraron en la literatura, los mejores resultados en Post-test FCI son de la Facultad de Ciencias Físicas y Matemáticas de la Universidad de Chile del grupo de solo hombres, que alcanzaron $79 \%$ en Post-test FCI con promedios en la prueba de entrada del FCI similares a Harvard University. Para las mujeres de esta universidad el promedio fue de $67,3 \%$ (Zamorano y Meza, [35,36]). En el caso de la Universidad de Chile, en las clases de cátedra se incorporaron las preguntas conceptuales del método de "Instrucción entre pares" (Peer Instruction; Mazur, [37]). Típicamente, se utilizaron dos preguntas de este tipo por clase para incentivar la discusión entre alumnos. Con esta misma finalidad, se mostraron alrededor de 40 videos cuidadosamente seleccionados de la "Video enciclopedia de demostraciones de Física" para ilustrar diversos experimentos de mecánica. Las clases fueron dictadas usando PowerPoint ${ }^{\circledR}$ y los apuntes de materia fueron publicados con anticipación para que los alumnos pudieran revisarlos antes de la clase. En las clases auxiliares se utilizó un esquema de trabajo cooperativo con problemas de contexto amplio [28,29]. Durante la última parte de la clase, los alumnos trabajaron en grupos, bajo la supervisión de los ayudantes, en la solución de uno o dos problemas de contexto amplio tomados del grupo de PER de la Universidad de Minnesota (http://groups.physics.umn.edu/physed). Meza y Zamorano [36] consideran que este esquema es ideal para los cursos grandes y produce, como se puede observar de la Fig.1, unos resultados excelentes. Aunque en algunos de los grupos de esta universidad a la eficiencia didáctica llegó a casi $48 \%$, los autores dicen que el desafío de ellos es llegar a $65 \%$ de la eficiencia didáctica del curso como se obtuvo en Harvard University.
Entre los años 2009 y 2011, de la Pontificia Universidad Católica de Perú han sido evaluados 4.535 alumnos con la prueba FCI (Castillo, [38]). Este grupo también se encuentra ubicado en la tercera elipse de la Figura 1 de los estudiantes con mejores puntajes de entrada, pero presenta una la eficiencia didáctica no mayor a $30 \%$. Los resultados indican que los conocimientos de fuerza y movimiento se implantan entre los cursos de Física 1 y Física 2, y no se encuentra una mejora significativa después. El concepto con mayor dificultad de aprendizaje es la segunda ley de Newton. Además, existe un importante número de alumnos para los cuales no hay relación entre la ganancia de conceptos y la aprobación del curso.

En Uruguay en la Universidad de la República, Facultad de Ingeniería, también fue utilizada el método que combina las estrategias de aprendizaje cooperativo con la resolución de problemas ricos en contexto [15] logrando porcentajes de Posttest FCI de $63 \%$ en promedio.

Finalmente, los puntos azules de Post-test $83 \%$ que están en la cuarta elipse de la Figura 1 son los resultados del Post-test de los estudiantes de Harvard University de Estados Unidos y de los profesores del Tecnológico de Monterrey de México, que participaron en los Cursos de Formación Profesional (Benegas, [39]). Aquí se dejaron estos datos como puntos de referencia de la meta a la cual deben llegar los estudiantes universitarios.

\section{Conclusiones}

En este artículo se presentaron los resultados del test FCI y sus aplicaciones para diferentes poblaciones universitarias de Estados Unidos, España y algunos países de América Latina. El análisis grafico permitió crear 4 grupos de universidades de acuerdo al nivel de conocimientos previos de los estudiantes que ingresan a la universidad: grupo "novatos", intermedio (donde existe la selección de los estudiantes), y dos grupos de nivel avanzados y "master" que son grupos de universidades de alto prestigio o de profesores de física. Al mismo tiempo se mostró como se puede, con los datos de pre-test y post-test FCI y la eficiencia didáctica, evaluar la efectividad de la enseñanza en contexto de las investigaciones actuales en América Latina. En especial, las mediciones realizadas con el FCI permiten decidir sobre la pertinencia del uso de una u otra estrategia didáctica al realizar una medición no sólo del nivel de maduración conceptual de cada alumno y por ende su preparación para afrontar retos superiores en sus carreras, sino que también permite medir la eficiencia didáctica que en últimas resulta ser una evaluación no tanto del alumno en sí, sino de la estrategia utilizada durante el desarrollo del curso para el desarrollo conceptual de los involucrados. La utilidad de este método puede ser extendida a otros campos conceptuales de la física u de otras ciencias exactas, ya que existen los test conceptuales de opción múltiple o cuestionarios con problemas para muchos temas de física, química y otras áreas del saber.

Con esta investigación la comunidad de educadores latinoamericanos tendrá un marco teórico propio para hacer las comparaciones entre las metodologías de enseñanza, entender la necesidad de mejoramiento de los planes de estudio y evaluar las necesidades de cambios curriculares. 
Tabla 1

Pre-test FCI, Post-test FCI y la eficiencia didáctica para diferentes grupos de estudiantes de diferentes países

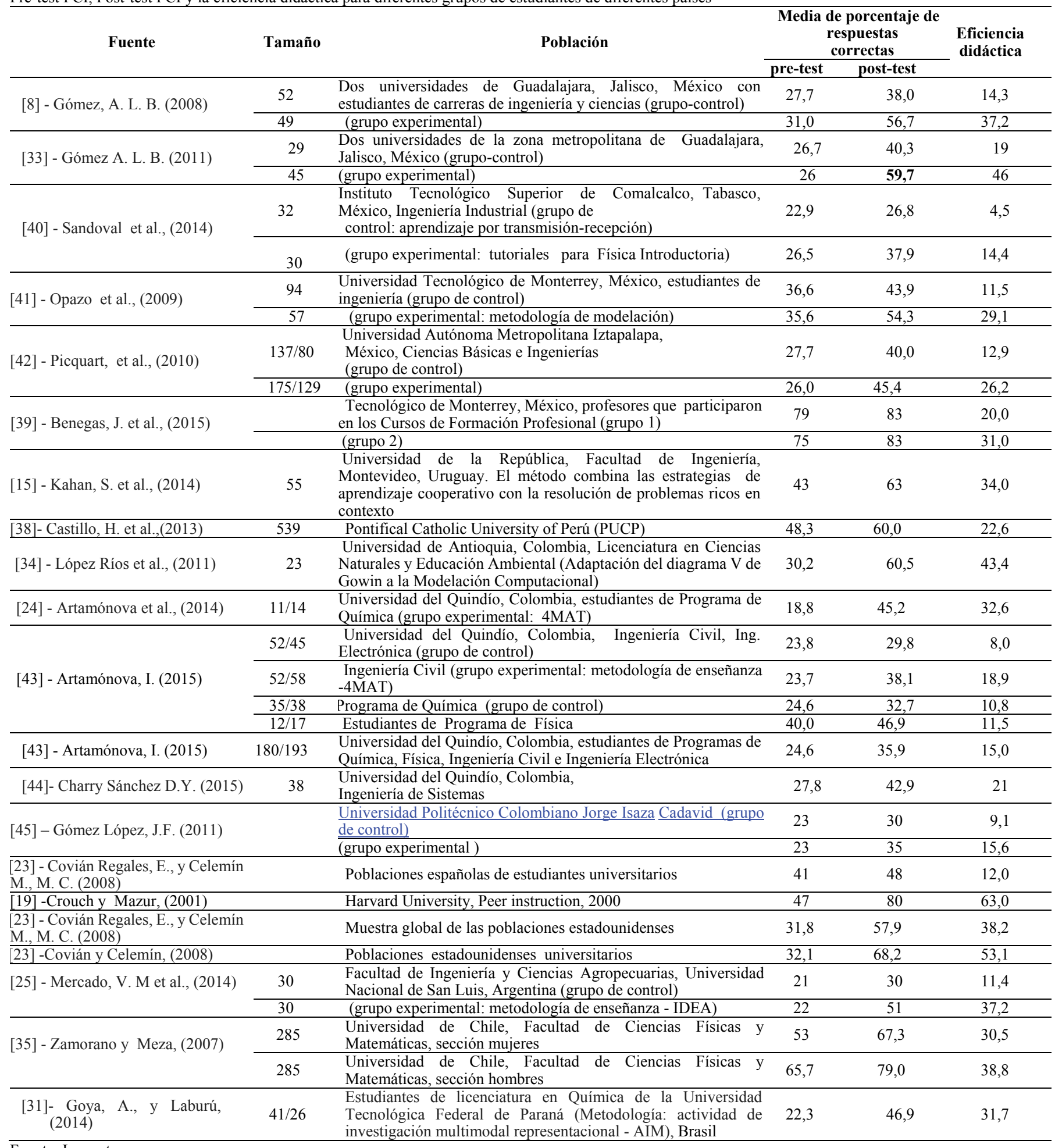

Fuente: Los autores

\section{Referencias}

[1] Furió, C. y Ortiz, E., Persistencia de errores conceptuales en el estudio del equilibrio químico. Enseñanza de las Ciencias, 1(1), pp.15-20, 1983.

[2] Hestenes, D. and Wells, M., A mechanics baseline test. The Physics Teacher, 30(3), pp. 159-166, 1992. DOI: 10.1119/1.2343498
[3] Hart, G.E. and Cottle, P.D., Academic backgrounds and achievement in college physics. The Physics Teacher, 31(8), pp. 470-475, 1993.

[4] Roeder, J.L. Physics appreciation versus Physics knowledge. The Physics Teacher, 36(6), pp.379-385, 1998. DOI: 10.1119/1.1527615

[5] Rodríguez-Martín, M.D., Mena-Romero, D.A. y Rubio-Atoche, C.M., Superación de errores conceptuales de estudiantes de ingeniería química 
industrial cuando estudian la asignatura de Física. Tecnología, Ciencia, Educación, pp. 40-46, ene.-jun. 2008.

[6] Mora, C. y Herrera, D., Una revisión sobre ideas previas del concepto de fuerza. Lat. Am. J. Phys. Educ., 3(1), pp.72-86, 2013.

[7] Cruz, M.I., Martínez, J.M.O., Sánchez, J.L. y Barbero, L.R., Detección de las ideas previas en cinemática utilizando la composición de movimientos. Investigación en la Escuela, 19, pp.105-118, 1993.

[8] Gómez, A.L.B., Acerca de la enseñanza-aprendizaje de los conceptos de fuerza y trabajo, Latin-American Journal of Physics Education, 2(3), pp. $18,2008$.

[9] McDermott, L.C. Concepciones de los alumnos y resolución de problemas en mecánica. [en línea]. 2009. Disponible en http://icar.univlyon2.fr/gric3/ressources/ICPE/espagno1/PartC/C1_chap p1-11.pdf,

[10] García, F.N.J., Calle, J.D.J.A. y Sánchez, J.J.V. Incidencia de la intervención didáctica en el aprendizaje de conceptos cinemáticos en estudiantes de Ingeniería de la UAM analizada desde sus ideas previas. Revista Educación en Ingeniería, 10(19), pp.26-38, 2015.

[11] Shaffer, P.S. and L.C. McDermott. Research as a guide for curriculum development: An example from introductory electricity. Part II: Design of an instructional strategy. American Journal of Physics, 60, pp. 10031013, 1992. DOI: $10.1119 / 1.16979$

[12] Thacker, B., Eunsook K., Kelvin, T. and Lea, S.M., Comparing problem solving performance of physics students in inquiry-based and traditional introductory physics courses. American Journal of Physics, 62, pp. 627633, 1994. DOI: $10.1119 / 1.17480$

[13] Driver, R., Psicología cognoscitiva y esquemas conceptuales de los alumnos. Enseñanza de las Ciencias, 4(1), pp.3-15, 1986.

[14] Aguilar, G.M. y Durán, D.M.F. Influencia de los dominios conceptuales en las competencias académicas: Área de física para ingeniería. Revista Educación en Ingeniería, 11(21), pp. 32-38, 2016.

[15] Kahan, S., Auyuanet, A., Davoine, F. y Stari, C., Física 1++: Aulas de aprendizaje cooperativo para estudiantes que recursan. Lat. Am. J. Phys. Educ. 8(2), pp.335-336, 2014.

[16] Hestenes, D., Wells, M. and Swackhamer, G., Force concept inventory. The Physics Teacher, 30, pp.141-158, 1992. DOI:10.1119/1.2343497

[17] Hestenes, D. and Halloun, I., Interpreting the force concept inventory. A response to Huffman and Heller. The Physics Teacher, 33, pp. 502-506, 1995. DOI: $10.1119 / 1.2344278$

[18] Hake, R., Interactive-engagement vs. traditional methods: A sixthousand-student survey of mechanics test data for introductory physics courses. Am. J. Phys., 66, pp. 64-74, 1998. DOI: 10.1119/1.18809

[19] Crouch, H. and Mazur, E., Peer instruction: Ten years of experience and results. American Journal of Physics, 69(9), pp. 970-977, 2001. DOI: $10.1119 / 1.1374249$

[20] Henderson, C., Common concerns about the Force Concept Inventory. The Physics Teacher, 40, pp. 542-547, 2002. DOI: 10.1119/1.1534822

[21] Caballero, M.D., Greco, E.F., Murray, E.R., Bujak, K.R., Marr, M.J., Catrambone, R. and Schatz, M.F., Comparing large lecture mechanics curricula using the Force Concept Inventory: A five thousand-student study. American Journal of Physics, 80(7), pp.638-644, 2012. DOI: $10.1119 / 1.3703517$

[22] Fagen, A.P., Crouch, C.H., and Mazur, E., Peer instruction: Results from a range of classrooms. The Physics Teacher, 40(4), pp.206-209, 2002. DOI: $10.1119 / 1.1474140$

[23] Covián, R.E. y Celemín, M.M.C. Diez años de evaluación de la enseñanza-aprendizaje de la mecánica de Newton en escuelas de ingeniería españolas. Rendimiento académico y presencia de preconceptos. Enseñanza de las Ciencias, 26(1), pp. 23-42, 2008.

[24] Artamónova, I., Mosquera, J.C., Ramírez, M.H. y Mosquera, J.D. Resultados cuantitativos de la aplicación del Sistema 4MAT en Mecánica en la Universidad del Quindío. Lat. Am. J. Phys. Educ. 8(4), pp. 4511-1-4511-8, 2014.

[25] Mercado, V.M., Alarcón, H., Benegas, J., Monasterolo, R.R., Rosales, F. and Ribotta, M.I.P.S.L., IDEA: An alternative for learning problem solving in the course of mechanics for engineering students at FICA. Journal of Education and Human Development, 3(4), pp.171-180, 2014, DOI: $10.15640 /$ jehd.v3n4a16.
[26] Beichner, J.D.D., GOAL-Oriented Problem Solving, [Online]. 2014. Available: https://lostcontact.mit.edu/afs/unity.ncsu.edu/lockers/ftp/beichner/RB/GOALPape r.pdf

[27] Panitz, E., Collaborative vs Cooperative learning: A comparison of the two concepts which will help us understand the underlying nature of interactive learning. [Online]. Available: http://files.eric.ed.gov/fulltext/ED448443.pdf

[28] Heller, P., Keith, R. and Anderson, S., Teaching problem solving through cooperative grouping. Part 1: Group versus individual problem solving, Am. J. Phys., 60, pp.627-636, 1992.

[29] Heller, P. and Hollabaugh, M., Teaching problem solving through cooperative grouping. Part 2: Designing problems and structuring groups, Am. J. Phys., 60, pp. 637-644, 1992.

[30] Leonard, W.J. y Dufresne, R.J., Resolución de problemas basada en el análisis. Hacer del análisis y del razonamiento el foco de la enseñanza de la física. Enseñanza de las ciencias: Revista de investigación y experiencias didácticas, 20(3), pp. 387-400, 2002.

[31] Goya, A. and Laburú, C.E., An experimental activity of physics by means of representational multimodal research. Experiências em Ensino de Ciências, 9(29, pp. 32-44, 2014.

[32] Laburú, C.E., De Freitas-Zompero, A. and Barros, M.A., Vygotsky e múltiplas representações: Leituras convergentes para o ensino de ciências. Caderno Brasileiro de Ensino de Física, 30(1), pp.7-24, 2013. DOI: $10.5007 / 2175-7941.2013 \mathrm{v} 30 \mathrm{n} 1 \mathrm{p} 7$

[33] Gómez, A.L.B., Un modelo de enseñanza neuropedagógico de las leyes de Newton para la Net Gen. Latin-American Journal of Physics Education, 5(2), pp. 33, 2011.

[34] López R.S., Veit, E.A. y Araujo, I.S., Modelación computacional apoyada en el uso del diagrama $\mathrm{V}$ de Gowin para el aprendizaje de conceptos de dinámica newtoniana. Revista Electrónica de Enseñanza de las Ciencias. 10(1), pp. 202-226, 2011.

[35] Zamorano, N. y Meza, A., Usando el cuestionario sobre conceptos de fuerza (FCI) como instrumento de diagnóstico y evaluación de $\begin{array}{llll}\text { aprendizajes, } & 2007 . & \text { [Online]. } & \text { Available: }\end{array}$ http://www.ici.ubiobio.cl/ccei2007/papers/43.pdf

[36] Meza, A. y Zamorano, N., El desafío de innovar en la enseñanza de la física: Ejemplo de una implementación exitosa basada en PER, 2008. [Online]. Available: http://www.ici.ubiobio.cl/ccei2007/papers/116.pdf

[37] Mazur, E., Peer instruction: A user's Manual, Universidad de Harvard, PEARSON, 1997, pp. 1-253.

[38] Castillo, H., Moscoso, R., Phan, J.L. y Quiroz, J., Impacto de la enseñanza de conceptos de fuerza y movimiento en los cursos de Física general. En Blanco y Negro, 4(1), 2013.

[39] Benegas, J., Alarcón, H. y Zavala, G., Formación de profesorado en metodologías de aprendizaje activo de la física, 2015. [Online]. Available

at: http://www.researchgate.net/profile/Genaro_Zavala/publication/264768 097_Formacin_de_Profesorado_en_Metodo logas_de_Aprendizaje_Activo_de_la_Fsica/links/53ee586d0cf26b9b7d c86a93.pdf

[40] Sandoval, M., Mora, C., Ramírez, M.H., Ricárdez, C. y De los Santos, U., Análisis del razonamiento conceptual en movimiento acelerado de estudiantes universitarios utilizando tutoriales de física introductoria. Lat. Am. J. Phys. Educ. 8(3), pp.573, 2014.

[41] Opazo, H.R.A., Eugenio, J. y Becerra, G., Influencia del razonamiento científico en el aprendizaje de conceptos en física universitaria: Comparación entre instrucción tradicional e instrucción por modelación, $2009 . \quad$ [Online]. Available at: http://www.comie.org.mx/congreso/memoriaelectronica/v10/pdf/area_t ematica_05/ponencias/1189 - F.pdf

[42] Picquart, M., Guzmán, O. y Sosa, R., Razonamiento científico e ideas previas en alumnos de ciencias básicas de la UAM-Iztapalapa. LatinAmerican Journal of Physics Education, 4(1), pp. 38, 2010.

[43] Artamónova, I., Aplicación del sistema 4MAT en la enseñanza de la mecánica: Caso particular de los estudiantes de programas de ingeniería civil y química en la Universidad del Quindío, Colombia, Ph.D. dissertation, CICATA-IPN, México D.F., México, 2015. 
[44] Charry-Sánchez, D.Y., Determinación de errores conceptuales de estudiantes de pregrado de la Universidad del Quindío que cursa el espacio académico Física I o Mecánica. Trabajo de Grado, Programa de Física, Universidad del Quindío, Armenia, Colombia, 2015.

[45] Gómez, L.J.F., Una propuesta de intervención didáctica que promueve el aprendizaje activo de la física universitaria y la utilización de un ambiente virtual interactivo de aprendizaje: Un estudio comparativo en el caso de la dinámica, MSc. Tesis, Dpto de Ciencias Sociales y Humanas, Universidad de Medellín, Medellin, Colombia, 2011. [Online]. Available at: http://cdigital.udem.edu.co/TESIS/CDROM61652011/11.Capitulo5.pdf

I. Artamónova, recibió el título de Físico y MSc. en Ciencias Físicas y Matemáticas en la Universidad Estatal de Moscú nombrada Lomonosov en 1991, Unión Soviética, título de MSc. en Enseñanza de las Matemáticas en 2004 en la Universidad Tecnológica de Pereira, Colombia, título de PhD en Física Educativa en 2015 en el Instituto Politécnico Nacional, CICATA, México. De 1992 hasta 2004 trabajo como contratista para la empresa privada, desde 2004 trabaja en el sector educativo. Desde 2008 es docente del Programa de Física de la Universidad del Quindío. Participó en proyectos internacionales y nacionales de investigaciones para: Organización Internacional de Migraciones, Ministerio de Relaciones Exteriores de Colombia, UN- INSTRAW International Research and Training Institute for the Advancement of Women de las Naciones Unidas, ASCUN - Asociación Colombiana de Universidades. Sus intereses investigativos incluyen temas de educación universitaria, migraciones internacionales, genero, aplicaciones estadísticas en diferentes áreas de las ciencias.

ORCID: 0000-0003-4584-4832

J.C. Mosquera-Mosquera, recibió el título de Físico, de MSc. en Ciencias Físicas y Matemáticas en 1991 y PhD en Física en 2008, todos en la Universidad Estatal de Moscú nombrada Lomonosov. Jefe de departamento de Aseguramiento de Calidad en 1993-1999, gerente de producción de una empresa en 1999- 2001. Desde 2001 docente de Física de la Universidad Tecnológica de Pereira, docente e investigador de la Universidad del Quindío desde 2003, actualmente director del Programa de Física de la Universidad del Quindío. Sus intereses investigativos incluyen temas de educación universitaria, procesamiento de imágenes por métodos acusto - ópticos, estadística aplicada

Scopus Author ID: 7006006502

ORCID: 0000-0001-7874-0736

J.D. Mosquera-Artamonov, recibió el título de Ing. Industrial de la Universidad Tecnológica de Pereira (2010), MSc. en Ingeniería de Calidad por la Universidad Autónoma de Querétaro (2014). Green Belt por la ASQ (2015). Magister en Ciencias en Ingeniería de Sistemas por la Universidad Autónoma de Nuevo León (2016). Estudiante doctoral en Ingeniería de Sistemas de la Universidad Autónoma de Nuevo León.

ORCID: 0000-0001-5419-6561 